\title{
Study of GaN-Based Light-Emitting Diodes Grown on Chemical Wet-Etching-Patterned Sapphire Substrate With V-Shaped Pits Roughening Surfaces
}

\author{
Ya-Ju Lee, Hao-Chung Kuo, Senior Member, IEEE, Tien-Chang Lu, Member, IEEE, \\ Shing-Chung Wang, Life Member, IEEE, Fellow, OSA, Kar Wai Ng, Kei May Lau, Fellow, IEEE, Zu-Po Yang, \\ Allan Shih-Ping Chang, and Shawn-Yu Lin
}

\begin{abstract}
We investigate the mechanism responding for performance enhancement of gallium nitride (GaN)-based light-emitting diode (LED) grown on chemical wet-etching-patterned sapphire substrate (CWE-PSS) with V-Shaped pit features on the top surface. According to temperature-dependent photoluminescence (PL) measurement and the measured external quantum efficiency, the structure can simultaneously enhance both internal quantum efficiency and light extraction efficiency. Comparing to devices grown on planar sapphire substrate, the threading dislocation defects of LED grown on CWE-PSS are reduced from $1.28 \times 10^{9} / \mathrm{cm}^{2}$ to $3.62 \times 10^{8} / \mathrm{cm}^{2}$, leading to a $12.5 \%$ enhancement in internal quantum efficiency. In terms of the theoretical computing of radiation patterns, the V-Shaped pits roughening surface can be thought of as a strong diffuser with paraboloidal autocorrelation function, increasing the escape probability of trapped photons and achieving a $20 \%$ enhancement in light extraction efficiency. Moreover, according to the measurement of optical diffraction power, CWE-PSS demonstrated superior guided light extraction efficiency than that of planar sapphire substrate, thus an extra $7.8 \%$ enhancement in light extraction efficiency was obtained. Therefore, comparing to the conventional LED, an overall $45 \%$ enhancement in integrated output power was achieved.
\end{abstract}

Index Terms-Epitaxial growth, light-emitting diodes (LEDs), optical device fabrication.

Manuscript received September 19, 2007; revised January 28, 2008. This work was supported by the MOE ATU program and in part by the National Science Council NSC 95-2120-M-009-008, NSC 95-2752-E-009-007-PAE, and NSC 95-2221-E-009-282, Republic of China. Dr. S.-Y. Lin would like to acknowledge the financial support of DOE-BES under grant DE-FG02-06ER46347 and Sandia National Laboratories.

Y.-J. Lee, H.-C. Kuo, T.-C. Lu, and S.-C. Wang are with the Department of Photonics, National Chiao Tung University, Hsinchu 300, Taiwan, R.O.C. (e-mail: yjlee.eo92g@nctu.edu.tw; hckuo@faculty.nctu.edu.tw; timtclu@ faculty.nctu.edu.tw; scwang@mail.nctu.edu.tw).

K. W. Ng and K. M. Lau are with the Photonics Technology Center, Department of Electrical and Electronic Engineering, The Hong Kong University of Science and Technology, Kowloon, Hong Kong, (e-mail: eekmlau@ust.hk).

Z.-P. Yang and S.-Y. Lin are with the Future Chips Constellation and Department of Physics, Applied Physics and Astronomy, Rensselaer Polytechnic Institute, Troy, NY 12180 USA (e-mail: sylin@rpi.edu).

A. S.-P. Chang is with the Molecular Foundry, Materials Science Division, Lawrence Berkeley National Laboratory, Berkeley, CA 94720 USA (e-mail: ASPChang@lbl.gov).

Color versions of one or more of the figures in this paper are available online at http://ieeexplore.ieee.org.

Digital Object Identifier 10.1109/JLT.2008.922151

\section{INTRODUCTION}

W ITH recent commercial success of GaN light-emitting diodes (LEDs), the need of even higher power and efficiency blue LEDs is imminent for their wide application in illumination [1]. These GaN LEDs were grown heteroepitaxially onto dissimilar substrates, such as sapphire and $\mathrm{SiC}$, because of difficulties in the growth of bulk GaN. Sapphire is the most commonly used substrate because of its relatively low cost. However, owing to the large mismatch of lattice constant and thermal expansion between epitaxial GaN films and sapphire substrates, $\mathrm{GaN}$ grown on planar sapphire substrates results in high density of dislocation defects $\left(10^{8}-10^{10} \mathrm{~cm}^{-2}\right)$ [2]. The threading dislocation (TD) defects originate from the interface between $\mathrm{GaN}$ and sapphire, and then thread into multiple quantum wells (MQWs) active region. It is known that the internal quantum efficiency $\left(\eta_{\text {Int }}\right)$ of LEDs is the ratio of the number of generated photons inside the MQWs to that of injected electron-hole pairs. With such an amount of TD defects mentioned above existing in GaN LEDs, energy states close to the middle of the energy gap would be generated, and a large portion of injected electron-hole pairs is trapped and recombined nonradiatively [3]. Therefore, $\eta_{\text {Int }}$ deteriorates due to the existence of TD defects. Furthermore, even when the injected electron-hole pairs recombine radiatively, most generated photons fall outside escape cones by total internal reflection due to the large refractive index difference between GaN and epoxy or air [4]. Thus, the light extraction efficiency $\left(\eta_{\text {Extraction }}\right)$ of LED devices is restricted as well. The external quantum efficiency $\left(\eta_{\text {Ext }}\right)$ is the ratio of the number of emitted photons outside the LED device to that of injected electron-hole pairs and is a useful physical parameter to evaluate LED performance. It is defined as [3]

$$
\eta_{\text {Ext }}=\eta_{\text {Int }} \cdot \eta_{\text {Extraction }}
$$

Thus, to enhance LED performance and alleviate issues mentioned above, there are various approaches to either improve $\eta_{\text {Int }}$ [5]-[9] or $\eta_{\text {Extraction }}$ [10]-[12]. However, few reports were published on enhancing LED performance by simultaneously improving $\eta_{\text {Int }}$ and $\eta_{\text {Extraction }}$ [13], [14].

In this paper, we report the detail investigation results of GaN LEDs grown on chemical wet etching-patterned sapphire substrate (CWE-PSS) with a roughening surface consisted of 
$\mathrm{V}$-shaped pits. The aim of this proposed structure is the reduction of TD defects by lateral overgrowth occurred on CWE-PSS and the increase of escape probability of emitting photons via the roughening surface. In Section II, we present the detail fabrication of three LED devices that are used for comparison in this study. The V-shaped pits roughening surface is investigated by using a scanning electron microscope (SEM) and transmission electron microscopy (TEM) in Section III. The paraboloidal autocorrelation function theoretically modeling the $\mathrm{V}$-shaped pits roughening surface is also given in this section and demonstrates that this roughening surface performs as a strong diffuser. In Section IV, we investigate the mechanism responding for improvement of the $\eta_{\text {Int }}$ of the LED grown on CWE-PSS. TEM is used to inspect the crystallography and TD defects of LED devices. The $\eta_{\text {Int }}$ of LEDs is measured by using temperature-dependent PL with variation of excitation power. In Section V, the optical diffraction of CWE-PSS is measured to study its efficiency as a trapped light extractor. The $\eta_{\text {Ext }}$ of three LEDs is measured, and the $\eta_{\text {Extraction of them is also }}$ derived based on the $\eta_{\text {Ext }}$ and $\eta_{\text {Int }}$ measured above. These results suggest that the LED grown on CWE-PSS combined with $\mathrm{V}$-shaped pits on top surface is a promising approach to dramatically enhance light output power of GaN LEDs.

\section{DEVICE FABRICATION}

The etched patterns on sapphire substrate are two dimensional hole-arrays arranged as hexagonal lattice. Here we use a mixed solution of sulfuric and phosphoric acids $\left(\mathrm{H}_{2} \mathrm{SO}_{4}: \mathrm{H}_{3} \mathrm{PO}_{4}=2: 1\right)$ to etch sapphire substrate at operating temperature of $300{ }^{\circ} \mathrm{C}$. The fabrication details of CWE-PSS can be found elsewhere [13]. Fig. 1(a) shows a top-view SEM image of CWE-PSS. The individual hole is $3 \mu \mathrm{m}$ in diameter and the lattice constant is $7 \mu \mathrm{m}$. The etched hole is $0.5 \mu \mathrm{m}$ in depth and has triangular-shaped $C$-plane in the center, surrounded by three $\{1-102\} R$-plane facets. The LED structure was then grown on CWE-PSS by low-pressure metal-organic-chemical-vapor deposition. Trimethylgallium (TMGa), trimethylindium (TMIn), and ammonia $\left(\mathrm{NH}_{3}\right)$ were used as the $\mathrm{Ga}$, In, and $\mathrm{N}$ precursors, respectively, while biscyclopentadienyl magnesium $\left(\mathrm{Cp}_{2} \mathrm{Mg}\right)$ and silane $\left(\mathrm{SiH}_{4}\right)$ were used as the p- and n-type doping sources. The device fabrication process is as follows. Following growth of a $\mathrm{GaN}$ nucleation layer at $520{ }^{\circ} \mathrm{C}$, a $1.5-\mu \mathrm{m}$ undoped $\mathrm{GaN}$ buffer layer and a $2.5-\mu \mathrm{m}$ Si-doped n-type $\left(5 \times 10^{17} \mathrm{~cm}^{-3}\right) \mathrm{GaN}$ cladding layer were grown at $1050{ }^{\circ} \mathrm{C}$. The active region consists of ten periods of 2.5-nm $\mathrm{In}_{0.23} \mathrm{Ga}_{0.77} \mathrm{~N} / 10-\mathrm{nm} \mathrm{GaN}$ MQWs structure with $\mathrm{Si}$ doping in $\mathrm{GaN}$ barriers and it was grown at $700{ }^{\circ} \mathrm{C}$. Beneath the MQWs structure is a strain-relaxation layer with five periods of 2.5-nm $\mathrm{In}_{0.1} \mathrm{GaN}_{0.9} \mathrm{~N} / 20-\mathrm{nm} \mathrm{GaN}$ superlattice (SL). Finally, a $0.4-\mu \mathrm{m} \mathrm{Mg-doped} \mathrm{p-type} \mathrm{GaN} \mathrm{cladding} \mathrm{layer} \mathrm{was} \mathrm{grown} \mathrm{on}$ top. Two different growth temperature of p-type GaN cladding layers, $1050{ }^{\circ} \mathrm{C}$ and $800{ }^{\circ} \mathrm{C}$, were used in this study. At a low growth temperature of $\mathrm{GaN}$ layers (i.e., $800{ }^{\circ} \mathrm{C}$ ), large number of $\mathrm{V}$-shaped pits can be seen on the top surface of the LED surface [15]. During the growth of these p-type cladding layers, the $\mathrm{Cp}_{2} \mathrm{Mg}$ flow rate was adjusted at different growth temperature so as to achieve the same $\mathrm{Mg}$ doping concentration of about $3 \times 10^{17} \mathrm{~cm}^{-3}$ in all these samples. The grown wafer (a)
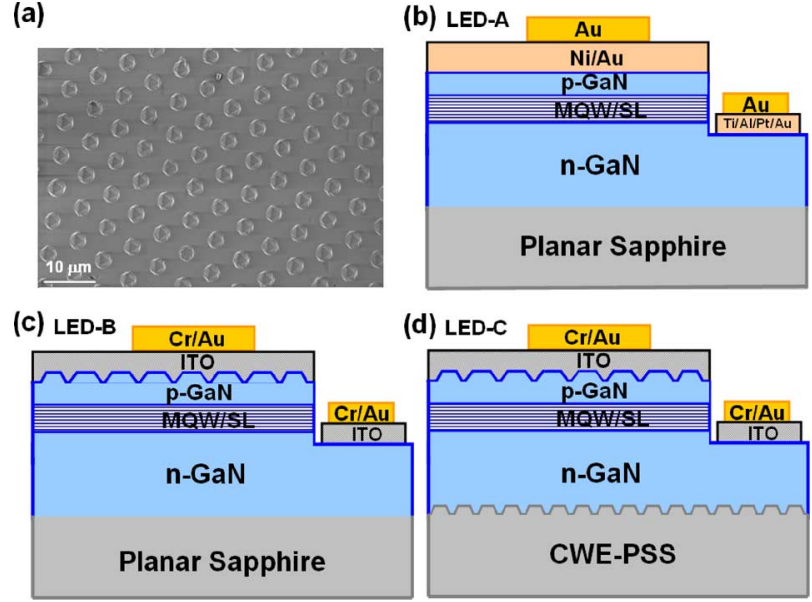

(d) LED-C Cr/Au

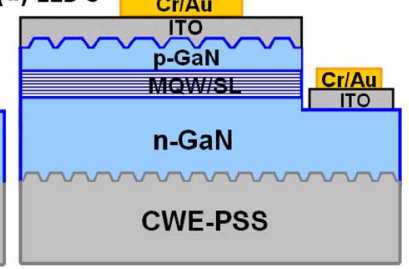

Fig. 1. (a) Top-view SEM image of CWE-PSS. (b), (c), and (d) show a schematic diagram of LED-A, LED-B, and LED-C, respectively.

was patterned with mesa size of $100 \times 180 \mu \mathrm{m}^{2}$ by a standard photolithographic process and partially dry-etched down to the n-type GaN. A 300-nm-thick indium-tin-oxide (ITO) was deposited as a current spreading layer and $\mathrm{Cr} / \mathrm{Au}$ were then deposited as $n$ and $p$ electrodes. For device comparison purpose, three different LED structures were fabricated. LED-A is the conventional LED, which was grown on planar sapphire substrate and has a flat surface morphology of p-type GaN grown on $1050{ }^{\circ} \mathrm{C}$. It shall be noted here that for LED-A, Ni-Au transparent contact layers were evaporated onto the $\mathrm{p}-\mathrm{GaN}$ cap layers to serve as the transparent contact layers (TCLs) and a thick Au layer was then deposited onto part of the TCL to serve as the p-electrodes. Ti-Al-Pt-Au contacts were deposited onto the exposed n-GaN layers to serve as the n-electrodes. LED-B was grown on planar sapphire substrate but has a roughened surface morphology of p-type $\mathrm{GaN}$ grown on $800^{\circ} \mathrm{C}$. LED-C was grown on CWE-PSS and also has a roughened surface morphology of p-type GaN grown on $800{ }^{\circ} \mathrm{C}$. Fig. 1(b)-(d) shows a schematic diagram of the final structure of LED-A, LED-B, and LED-C, respectively.

\section{V-Shaped Pits Roughening Surface}

The SEM was used to investigate the change of surface morphology of p-type GaN caused by growth temperature. Fig. 2 shows SEM images of surface morphology of LED-A, LED-B, and LED-C. In Fig. 2(a), it is clearly seen that the surface of LED-A is quite flat and contains very few hexagonal pits. These hexagonal pits are originating from the so called V-shaped pit in MQWs [16]-[18]. The strain induced by the lattice mismatch between $\mathrm{InGaN}$ and $\mathrm{GaN}$ layers and the reduction of surface mobility due to the relative low growth temperature $\left(700^{\circ} \mathrm{C}\right)$ in MQWs acts as a driving force for the formation of the $\mathrm{V}$-shaped pits in MQWs. The formation of V-shaped pits in MQWs is mainly to release high strain energy induced by heteroepitaxy of InGaN/GaN materials around TD defects. Thus, the bottom of each V-shaped pit was always connected to a TD defect, and the density of $\mathrm{V}$-shaped pits in MQWs is almost equivalent to that of TD defects [16]. However, the subsequent growth of p-type $\mathrm{GaN}$ at $1050{ }^{\circ} \mathrm{C}$ completely fills the $\mathrm{V}$-shaped pits in MQWs 

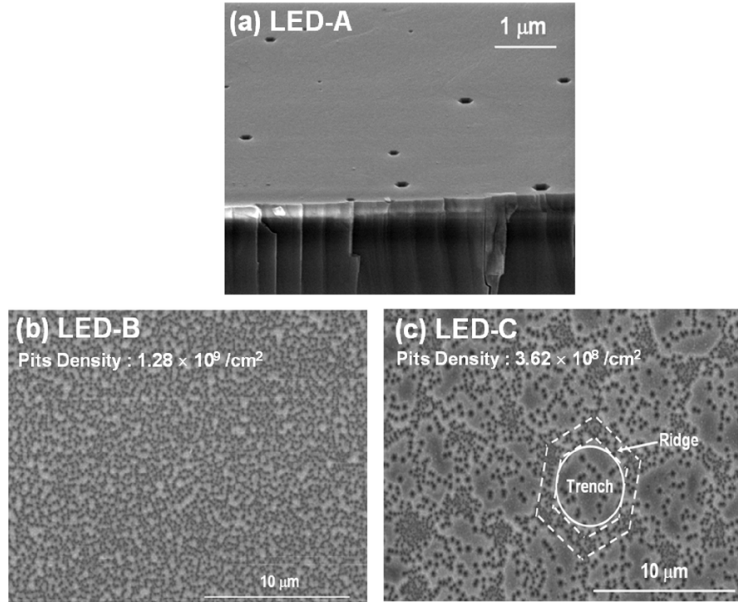

Fig. 2. Surface morphology SEM images of (a) LED-A, (b) LED-B, and (c) LED-C. Comparing (a) to (b), a large number of V-shaped pits were observed on the top surface of LED-B. In LED-C, most V-shaped pits (within hexagon mark) were observed in ridge regions of hole-arrays of CWE-PSS.

due to the sufficient surface mobility provided under such high growth temperature. Therefore, a flat p-type $\mathrm{GaN}$ surface was observed in Fig. 2(a). For LED-B and LED-C, during growth of p-type $\mathrm{GaN}$, we reduce the surface mobility of migrated $\mathrm{Ga}$ atoms by decreasing growth temperature to $800{ }^{\circ} \mathrm{C}$. Due to the insufficient energy for $\mathrm{Ga}$ atoms to migrate to proper sites, the lateral growth rate of $\mathrm{GaN}$ will become smaller than vertical growth rate of $\mathrm{GaN}$ and the $\mathrm{V}$-shaped pits profile was conformally formed in p-type GaN as that in MQWs. Fig. 3(a) shows a cross-sectional TEM image of LED-B. In Fig. 3(a), lots of $\mathrm{V}$-shaped pits in MQWs were observed and most bottoms of pits were connected to TD defects, as mentioned before. In Fig. 3(a), apex positions of $\mathrm{V}$-shaped pits in MQWs and p-type GaN are almost the same. As further evidence, an enlarged cross-sectional TEM image focusing on MQWs of LED-B is shown in Fig. 3(b). The apexes of both pits in MQWs and p-type GaN were penetrated and connected by TD defects. Therefore, for LED-B and LED-C, densities of V-shaped pits in the very top surface of p-type GaN are almost equivalent to that of TD defects. In this paper, we applied above approach to fabricate a roughening surface and account for the density of TD defects for LED-B and LED-C. In Fig. 2(b), the V-shaped pits are distributed randomly on the top surface of LED-B, and its surface density was estimated to be $1.28 \times 10^{9} / \mathrm{cm}^{2}$.

However, in Fig. 2(c), the V-shaped pits are distributed mainly in ridge regions (outside hole-arrays patterns of
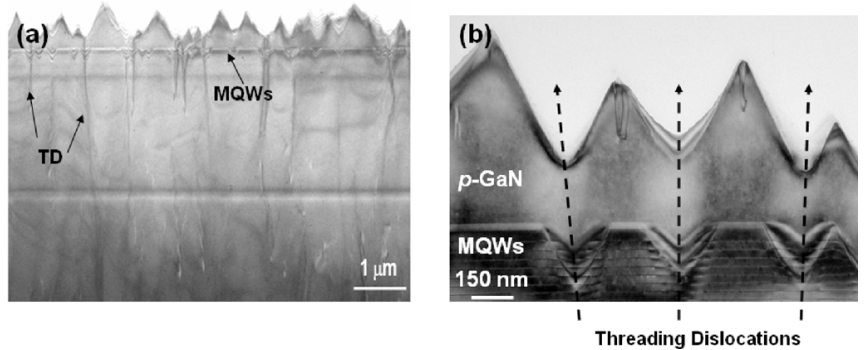

Fig. 3. (a) Cross-sectional TEM image of LED-B. (b) Enlarged cross-sectional TEM image focusing on MQWs of LED-B.

CWE-PSS) and its density dramatically decreases in trench regions (within hole-arrays patterns of CWE-PSS).

Thus for LED-C, the overall surface density of $\mathrm{V}$-shaped pits is $3.62 \times 10^{8} / \mathrm{cm}^{2}$. It was 3.5 times smaller than that of LED-B, indicating a significant reduction of TD defects. We believe the reduction of TD defects is due to adopting CWE-PSS scheme. The detail will be explained later.

To examine the diffused power of $\mathrm{V}$-shaped pits in p-type $\mathrm{GaN}$, angular dependent radiation pattern is measured using a $\mathrm{He}-\mathrm{Ne}$ laser and the result is shown in Fig. 4(a). The He-Ne laser is incident normally from the backside (sapphire) of LED-B wafer and the transmitted power was measured from $-80^{\circ}$ to $80^{\circ}$, as shown in the insert of Fig. 4(a). The measured result of LED-A wafer is also plotted in Fig. 4(a) for comparison. The calculated radiation pattern of LED-B wafer is also shown in Fig. 4(a) and is given by the paraboloidal autocorrelation function describing the wide angle scattering of $\mathrm{V}$-shaped pits [19] [see (2), shown at the bottom of the page], where $\lambda$ is the incident wavelength, $\theta_{0}$ is the incident angle of emitting light, $\theta$ is the detected angle, and $\omega$ is the correlation length of the roughening surface. $\mathrm{S}$ is the rms phase delay and defined as

$$
S\left(\theta_{0}\right)=2 \pi \frac{\sigma_{h}}{\lambda}\left(\sqrt{n^{2}-\sin ^{2} \theta_{0}}-\cos \theta_{0}\right) .
$$

where $\sigma_{h}$ is the rms roughness of roughening surface and $n$ is the index of refraction of $\mathrm{GaN}$ material. According to (2), the diffused behavior of $\mathrm{V}$-shaped pits roughening surface corresponds to a paraboloidal autocorrelation function for the largest roughness component $\left(\mathrm{S}_{1}\right.$ and $\left.\omega_{1}\right)$ with an exponential autocorrelation function for the second roughness component $\left(\mathrm{S}_{2}\right.$ and $\left.\omega_{2}\right)$ [19]. The V-shaped pits roughening surface can be described perfectly with $\mathrm{S}_{2}=0.26, \omega_{2} / \lambda=1.7$, and $\omega_{1} / \mathrm{S}_{1} \lambda=0.31$ in (2), as shown in Fig. 4(a).

$$
\begin{aligned}
\langle I\rangle=\cos \theta \cdot \pi \cdot \exp \left(-S_{2}^{2}\right) \cdot & {\left[\left(\frac{\omega_{1}}{\lambda S_{1}}\right)^{2} \cdot \exp \left\{-\left(\frac{\pi \omega_{1}}{\lambda S_{1}}\right)^{2} \cdot\left[\sin ^{2} \theta-2 \sin \theta \sin \theta_{0}+\sin ^{2} \theta_{0}\right]\right\}\right.} \\
& \left.+2\left(\frac{S_{2} \omega_{2}}{\lambda}\right)^{2}\left\{1+\left(\frac{2 \pi \omega_{2}}{\lambda}\right)^{2} \cdot\left[\sin ^{2} \theta-2 \sin \theta \sin \theta_{0}+\sin ^{2} \theta_{0}\right]\right\}^{-3 / 2}\right]
\end{aligned}
$$




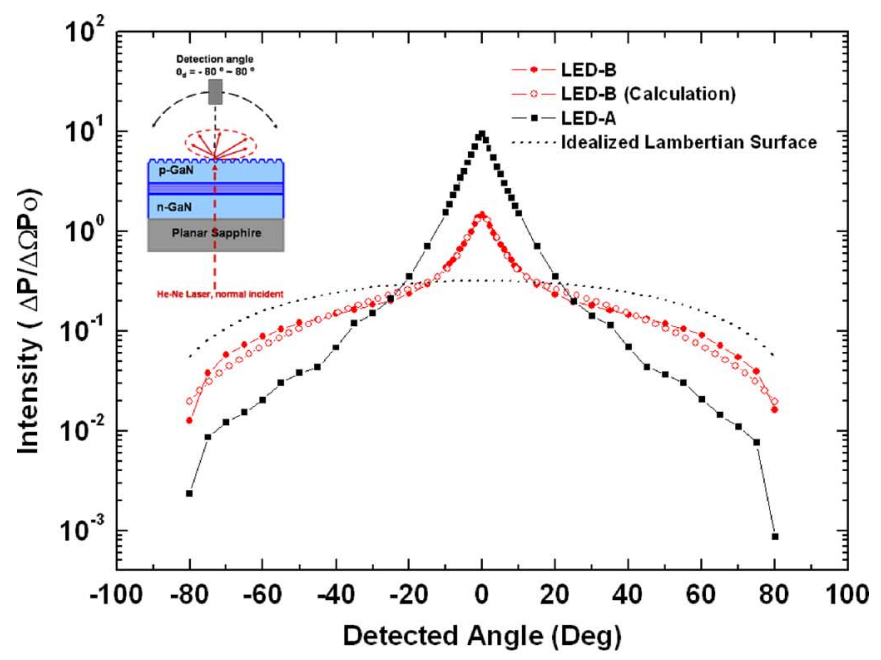

(a)

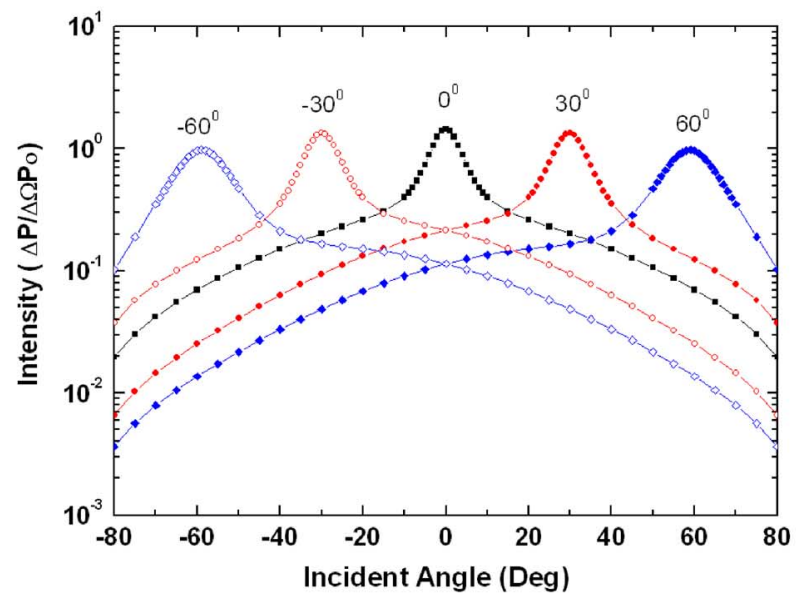

(b)

Fig. 4. (a) Angular-dependent radiation pattern of flat surface (LED-A) and V-shaped pits roughening surface (LED-B) measured by using $\mathrm{He}-\mathrm{Ne}$ laser beam at normal incidence. The radiation pattern of ideal Lambertian diffuser is also plotted for comparison (dotted line). The theoretical fitting of radiation pattern of LED-B is calculated using paraboloidal autocorrelation function. (b) Calculated radiation patterns of $\mathrm{V}$-shaped pits roughening surface emitted by light with incident angle $\left(\theta_{0}\right)$ of $0^{\circ}, \pm 30^{\circ}$, and $\pm 60^{\circ}$.

It is also useful to compare the radiation patterns of LED-A and LED-B with that of idealized Lambertian diffuser, that is given by

$$
\langle I\rangle=\frac{\cos \theta}{\pi}
$$

In Fig. 4(a), the diffused power of LED-B is between that of idealized Lambertian diffuser and LED-A. At detected angle of 0 degree, the detected power of LED-B is one order of magnitude smaller than that of LED-A. On the other hand, at large detected angle $\left(|\theta|>25^{\circ}\right.$, the detected power of radiation pattern of LED-B is almost one order larger than that of LED-A. That means the roughening surface of LED-B could diffuse light emitted from MQWs efficiently. The diffused surface is very useful for the consideration of photon extraction [11], [12]; especially for GaN LEDs that the critical angle is only about $\sin ^{-1}(1 / 2.4) \sim 25^{\circ}$. To predict diffused radiation patterns of

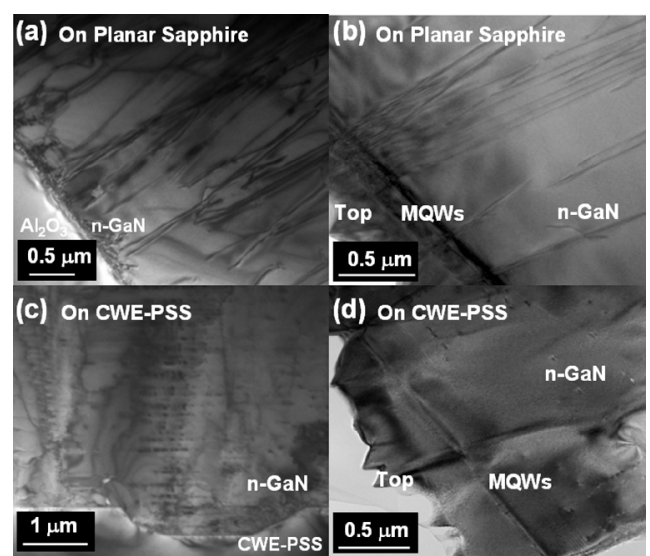

Fig. 5. Cross-sectional TEM images of GaN/sapphire interface grown on (a) planar sapphire substrate (LED-B) and (c) CWE-PSS (LED-C). Cross-sectional TEM images of MQWs region grown on (b) planar sapphire substrate (LED-B) and (d) CWE-PSS (LED-C).

$\mathrm{V}$-shaped pits emitted by light with the angle larger than the critical angle, $\theta_{0}= \pm 30^{\circ}$ and $\pm 60^{\circ}$ are substituted in (2) and the results is shown in Fig. 4(b). In Fig. 4(b), strong diffused power is still observed, even though the incident angles of emitting light are larger than the critical angle, indicating that the $\mathrm{V}$-shaped pits could be thought as a strong diffuser of guided light.

\section{INTERNAL QUANTUM EFFICIENCY}

Now we focus on investigations of the reduction of TD defects density and the improvement of $\eta_{\text {Int }}$ for LED-C grown on CWE-PSS. To shed light on the origin of $\eta_{\text {Int }}$ enhancement by CWE-PSS, we used TEM to investigate crystalline quality of LEDs grown on CWE-PSS (LED-C) and on planar sapphire substrate (LED-B). For the LED grown on planar sapphire substrate (LED-B), cross-sectional TEM images of the GaN/ sapphire interface and MQWs region are shown in Fig. 5(a) and (b), respectively. Bunches of dislocation defect threads can be seen radiating vertically from the GaN/sapphire interface into the MQWs region. The crystallography of films on CWE-PSS (LED-C) is drastically different as depicted in Fig. 5(c) and (d), which shows cross-sectional TEM images of GaN/ sapphire interface and MQWs region of the LED grown on CWE-PSS (LED-C), respectively. As shown in Fig. 5(c), a large number of stacking faults are present in the trench region. The formation of stacking faults could be due to the homoepitaxial lateral growth initiated from the ridge region to cover the trench region during the initial growth of GaN buffer layer, leading to high crystalline quality over trench regions [20]. These stacking faults interact with the vertical threading dislocations and bend them horizontally [21]. Thus, fewer threading dislocations can penetrate into the active regions, implying high crystalline quality of LED-C grown on CWE-PSS. This is also the reason why fewer $\mathrm{V}$-shaped pits were observed on LED-C within trench regions of hole-arrays patterns, as shown in Fig. 2(c).

Besides, according to Fig. 5(d), the cross section becomes clearer in region close to the MQWs, indicating the reduction of stacking faults density there. Thus, the stacking faults will 


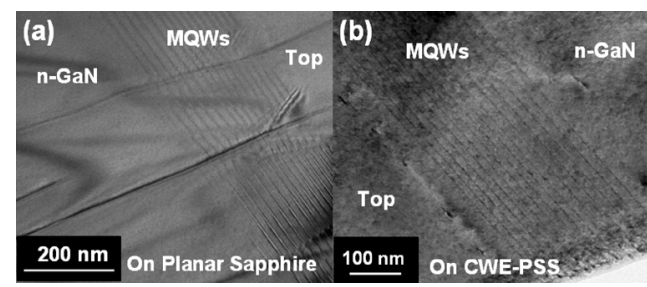

Fig. 6. Enlarged TEM images of the SL/MQWs region of LED grown on (a) planar sapphire substrate (LED-B) and (b) CWE-PSS (LED-C).

not have negative impact for active region because they terminate beneath the MQWs. As further evidence, Fig. 6(a) and (b) show enlarged TEM images of the SL/MQWs region of LED grown on planar sapphire substrate and CWE-PSS, respectively. In Fig. 6(a), the threading dislocation defects obviously penetrate through the SL/MQWs region into the upper p-type GaN cladding layer. These penetrating defects induce leakage paths for injected carriers that decrease internal quantum efficiency of LED device. In stark contrast, clear interfaces between well and barrier in MQWs are observed in Fig. 6(b) without any interruption of threading dislocation defects. Therefore, for LED grown on CWE-PSS, injected carriers can recombine and generate light more efficiently in active region, as compared to LED grown on planar sapphire substrate.

To further investigate the improvement of $\eta_{\text {Int }}$ for LED-C grown on CWE-PSS, the temperature-dependent PL was measured with varying of excitation laser power. Excitation-dependent PL measurement was carried out for LED-B and LED-C at 300 and $10 \mathrm{~K}$ by $\mathrm{He}-\mathrm{Cd}$ laser $(325 \mathrm{~nm}, 50 \mathrm{~mW}$ ). PL quantum efficiency was calculated by [22]

$$
\eta_{\mathrm{PL}}=C \frac{\frac{I_{\mathrm{PL}}}{E_{\mathrm{PL}}}}{\frac{I_{\mathrm{EX}}}{E_{\mathrm{EX}}}}
$$

where $I_{\mathrm{PL}}$ and $I_{\mathrm{EX}}$ are PL and excitation intensities, respectively. $E_{\mathrm{PL}}$ and $E_{\mathrm{EX}}$ are PL photon energy and excitation photon energy, respectively. $C$ is a constant affected by carrier injection efficiency by laser, light extraction and correction efficiency of PL, and does not depend on either excitation power density or measurement temperature. Fig. 7(a) and (b) shows relative PL quantum efficiency for LED-B and LED-C, respectively. Curves are normalized by the peak value at 10 $\mathrm{K}$ for both samples. As can be seen, the efficiency is strongly dependent on excitation power. Basically, light extraction efficiency does not depend on either injected carriers or temperature. Therefore, the efficiency curves measured at different temperatures in Fig. 7 infer $\eta_{\text {Int }}$ of LED devices. According to Fig. 7, $\eta_{\text {Int }}$ at the injection carrier density of $1 \times 10^{20} \mathrm{~s}^{-1} \mathrm{~cm}^{-2}$ are $\sim 56 \%$ and $63 \%$ for LED-B and LED-C, respectively. Comparing LED-B and LED-C, around $12.5 \%$ enhancement in $\eta_{\text {Int }}$ is due to the better crystalline quality of LED-C grown on CWE-PSS. It should be noted that both LED-A and LED-B were grown on planar sapphire substrate and the epitaxial structures are identical except for the growth temperature of p-type GaN. Basically, $\eta_{\text {Int }}$ of LED is dominated by the crystalline quality and defect density in the MQWs, and the subsequent growth of p-type GaN above MQWs does not affect

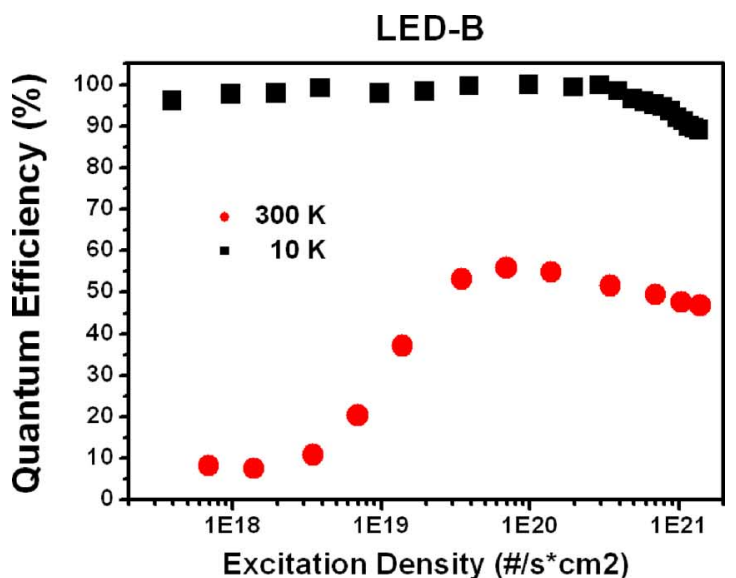

(a)

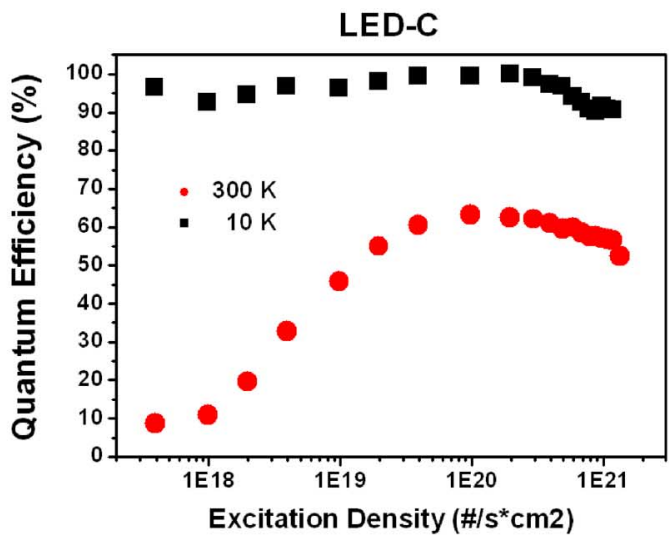

(b)

Fig. 7. Relative PL quantum efficiency as a function of excitation power for LEDs grown on (a) planar sapphire substrate (LED-B) and (b) CWE-PSS (LED-C) measured at 10 and $300 \mathrm{~K}$.

$\eta_{\text {Int }}$ much. Besides, in this article we use PL to evaluate the enhancement of IQE contributed from CWE-PSS, i.e., the pumping light is directly emitted into MQWs and subsequently absorbed to produce electron-hole pairs. The electron-hole pairs then recombine radiatively. In this case, the difference of resistivity due to the incorporation or doping efficiency of $\mathrm{Mg}$ in p-type $\mathrm{GaN}$ grown at different temperatures would not affect measurement of IQE much. Therefore, it is reasonable that we assume $\eta_{\text {Int }}$ of LED-A is same as that of LED-B, or is $\sim 56 \%$. In addition to enhance $\eta_{\text {Int }}$, CWE-PSS can improve $\eta_{\text {Extraction }}$ as well.

\section{LIGHT EXTRACTION EFFICIENCY}

To explain the enhancement in light extraction by using CWE-PSS, far-field optical diffraction by the CWE-PSS is measured using a He-Ne laser (beam spot size 2-mm) in experimental setup depicted in Fig. 8(a). The incident angle $\theta_{i}$ of the $\mathrm{He}-\mathrm{Ne}$ laser is fixed at $50^{\circ}$ and the detected angle $\theta_{d}$ is varied from $-80^{\circ}$ to $80^{\circ}$. The measured angular spectrum of diffracted power by CWE-PSS is shown in Fig. 8(b). The inset in Fig. 8(b) is Fraunhofer diffraction pattern of CWE-PSS recorded at a distance of $40 \mathrm{~cm}$ from the sample. The brightest dot in the center of diffraction pattern corresponds to the principal maxima of diffraction, which is located at $\theta_{d}=-50^{\circ}$. 
(a)

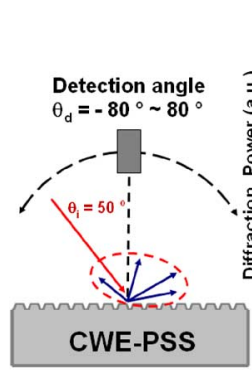

(b)

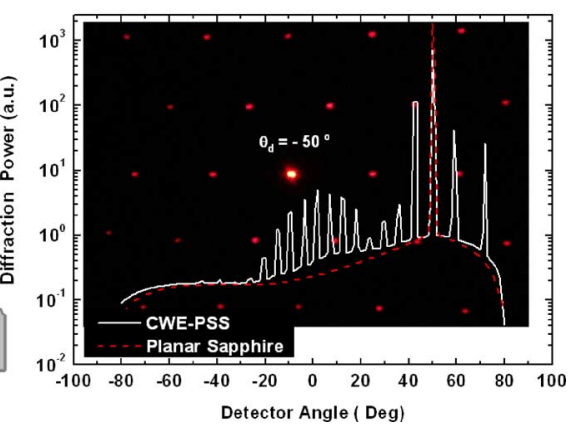

Fig. 8. (a) Experimental setup for measuring optical diffraction by CWE-PSS. (b) Measured angular dependence of diffraction power by CWE-PSS; inset (background) in Fig. 8(b) is measured Fraunhofer diffraction patterns of CWE-PSS

Considering the grating equation, the relation between incident wavelength $\lambda$, angle of incidence $\theta_{i}$, and angle of reflection of the $m$ th order $\theta_{R}^{(m)}$ is given by [23]

$$
m \lambda=d\left(\sin \theta_{i}+\sin \theta_{R}^{(m)}\right)
$$

where $m$ is the order of diffraction and $d(7 \mu \mathrm{m})$ is separation between two identical slits. We calculate $\theta_{R}^{(m)}$ of all different orders by using (6) and find them to be consistent with the measured results in Fig. 8(b). Thus, CWE-PSS serves the function as a diffraction grating. The refractive index of GaN $\left(n_{\mathrm{GaN}}\right)$ and sapphire $\left(n_{\text {sapphire }}\right)$ are 2.4 and 1.7 , respectively. The critical angle of GaN/sapphire interface is equal to $\sin ^{-1}(1.7 / 2.4) \sim$ $45^{\circ}$ and that of GaN-air interface is equal to $\sin ^{-1}(1 / 2.4) \sim$ $25^{\circ}$. Thus, light emitted from MQWs with incident angle of $50^{\circ}$ at either interface will be completely trapped inside the LED device and only light with incident angle within $\pm 25^{\circ}$ can be extracted from GaN-air interface. From Fig. 8(b), the total integrated diffraction power from CWE-PSS within the escape cone from $-25^{\circ}$ to $25^{\circ}\left(\boldsymbol{P}_{\mathrm{CWE}-\mathrm{PSS}}\right)$ on the plane of incidence is found to be ten times larger than that from planar sapphire substrate $\left(\boldsymbol{P}_{\text {planar }}\right)$. Therefore, in addition to reducing the density of TD defects, CWE-PSS also serves as a diffraction grating that effectively diffracts a portion of guided-light into the escape cone and increases escape probability of photons inside LED chip. It shall be noted that in real case, light propagates in GaN material, thus the optical wavelength $\left(\lambda / n_{\mathrm{GaN}}\right)$ is smaller than that in the air. Therefore, the smaller $\theta_{R}^{(m)}$ is obtained in (6), indicating larger enhancement is expected since the integrated diffracted power within $\pm 25^{\circ}$ increases as well.

Fig. 9(a) and (b) shows an optical microscope image of LED-B and LED-C operating at $1 \mathrm{~mA}$. The corresponding EL intensity distributions of LED-B and LED-C are shown in Fig. 9(c) and (d), respectively. The EL intensity observed from the LED-C clearly exceeded those from LED-B. It can be seen that there are many localized light-emitting dots on LED-C. According to Fig. 9(a), these light-emitting dots locate in the trench region of CWE-PSS, indicating stronger EL efficiency in this region. For LED-B, its EL intensity distribution is relative uniform, without observable any localized light-emitting dots.

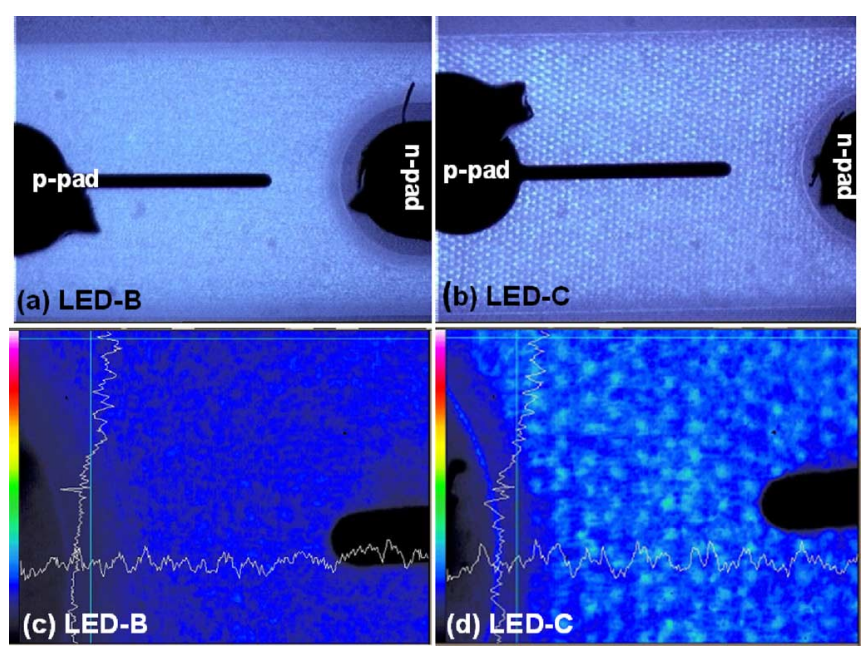

Fig. 9. Optical microscope images of (a) LED-B and (b) LED-C operating at $1 \mathrm{~mA}$. EL intensity distributions of (c) LED-B and (d) LED-C.

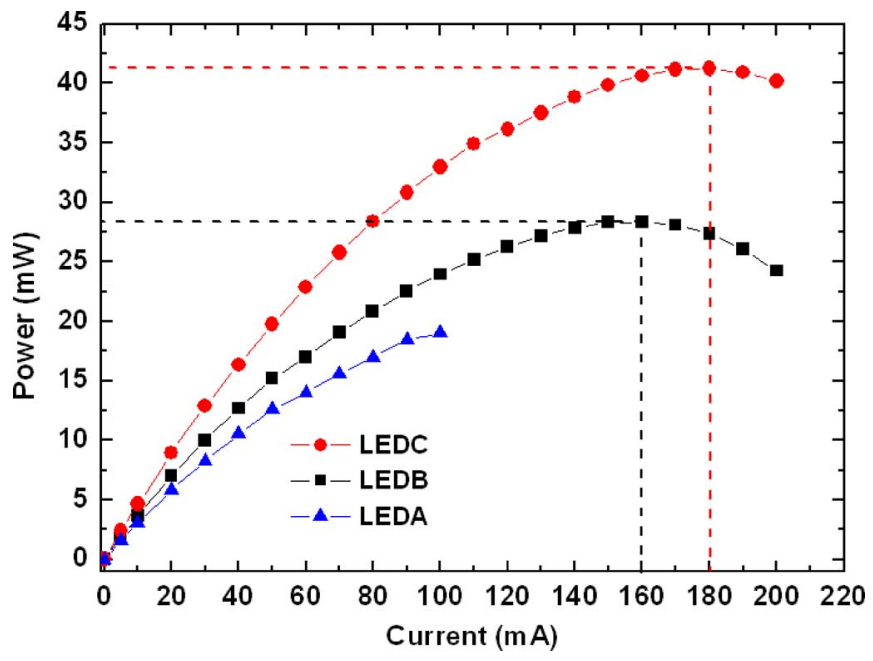

Fig. 10. Integrated output optical power versus drive current ( $L-I$ curve) of LED-A, LED-B, and LED-C.

The higher light-emitting dots emitting from the trench regions in LED-C is due to good crystalline quality in this region. In addition, such an enhancement could also be attributed as a consequence of the enhanced $\eta_{\text {Extraction }}$ of LED-C grown on CWE-PSS compared to LED-B grown planar sapphire substrate, due to the effect of optical diffraction from CWE-PSS as mentioned above.

The integrated output optical power versus drive current $(L-I$ curve) of LED-A, LED-B, and LED-C is shown in Fig. 10. The LED-C, grown on CWE-PSS and with V-shaped pits on top surface, produced much higher light output as compared with that of LED-A and LED-B under all our measurement condition. With injection current of $20 \mathrm{~mA}$, it was found that electroluminescence (EL) peaks all occurred at around $465 \mathrm{~nm}$ for these LEDs since exactly the same MQW structure was used. With a 20-mA current injection, it was found that output powers without epoxy resin encapsulant were $5.8,7.0$, and $8.5 \mathrm{~mW}$ for LED-A, LED-B, and LED-C, respectively. The saturated current, defined as the current corresponding to the saturated output power, of LED-C is $20 \mathrm{~mA}$ larger than that of LED-B. It is 
TABLE I

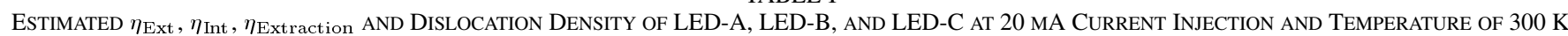

\begin{tabular}{cccc}
\hline Substrate & LED-A & LED-B & LED-C \\
\hline $\begin{array}{c}\text { Measured External quantum } \\
\text { efficiency. after package }\left(\eta_{\text {Ext }}\right)\end{array}$ & $27.5 \%$ & $33 \%$ & $40 \%$ \\
$\begin{array}{c}\text { Measured internal quantum } \\
\text { efficiency }\left(\eta_{\ln }\right)\end{array}$ & $56 \%$ & $56 \%$ & $63 \%$ \\
$\begin{array}{c}\text { Calculated light extraction } \\
\text { efficiency }\left(\eta_{\text {Extraction }}\right)\end{array}$ & $49.1 \%$ & $58.9 \%$ & $63.5 \%$ \\
\begin{tabular}{c} 
Dislocation Density $\left(/ \mathrm{cm}^{2}\right)$ \\
\hline
\end{tabular} & $1.28 \times 10^{9}$ & $1.28 \times 10^{9}$ & $3.62 \times 10^{8}$ \\
\hline
\end{tabular}

because by adopting CWE-PSS scheme, more valid active regions without penetration of leakage paths are injected by carriers. Comparing LED-B to LED-A at $20 \mathrm{~mA}$, about $20 \%$ enhancement of output power was observed. As mentioned above, the $\eta_{\text {Int }}$ of LED-A and LED-B are identical ( 56\%), thus the main enhancement of output power is mainly attributed to higher $\eta_{\text {Extraction }}$ benefiting from $\mathrm{V}$-shaped pits. Comparing the output power of LED-B to that of LED-C at $20 \mathrm{~mA}$, LED-C demonstrates $\sim 20 \%$ enhancement over LED-B. The enhancement is attributed not only to high crystalline quality in MQWs, but also to strong diffraction by CWE-PSS.

The external quantum efficiency $\left(\eta_{\text {Ext }}\right)$ after package can be derived from $L-I$ curve and its corresponding emitting wavelengths. Thus, based on these measured $\eta_{\text {Int }}$ and $\eta_{\text {Ext }}$, light extraction efficiency ( $\left.\eta_{\text {Extraction }}\right)$ can be estimated. At an injection current of $20 \mathrm{~mA}, \eta_{\text {Exe }}, \eta_{\text {Int }}$, and $\eta_{\text {Extraction }}$ for LED-A, LED-B, and LED-C are summarized in Table I. The densities of TD defects for three samples are also listed in Table I. For LED-A, the density of TD defects is estimated by counting for etching pits density and it is the same as that of LED-B [24]. According to Table I, the $\eta_{\text {Ext }}$ are estimated to be $\sim 27.5 \%, 33 \%$, and $40 \%$ for LED-A, LED-B, and LED-C, respectively. Comparing LED-B to LED-A, the main enhancement of $\eta_{\mathrm{Ext}}$ is due to the $20 \%$ enhancement of $\eta_{\text {Extraction, benefiting from the roughening surface of }}$ $\mathrm{V}$-Shaped pits, as mentioned before. Comparing LED-C to LED-B, 12.5\% and 7.8\% enhancements were observed in $\eta_{\text {Int }}$ and $\eta_{\text {Extraction }}$, respectively; thus $\sim 20 \%$ overall enhancement was obtained in $\eta_{\text {Ext }}$. The main enhancements in $\eta_{\text {Int }}$ and $\eta_{\text {Extraction }}$ are attributed to the reduction of TD density and the strong diffraction from CWE-PSS, respectively. Comparing LED-C to LED-A, 12.5\% and $29 \%$ enhancements were observed in $\eta_{\text {Int }}$ and $\eta_{\text {Extraction }}$, respectively; thus an overall $45 \%$ enhancement was obtained in $\eta_{\text {Ext }}$. Therefore, comparing to conventional LEDs, the LED structure adopting CWE-PSS and V-shaped pits on top surface can simultaneously enhance $\eta_{\text {Int }}$ and $\eta_{\text {Extraction }}$, resulting in a dramatic improvement of total output power.

\section{SUMMARY}

In summary, the crystallography and optical characteristics of GaN-based LED grown on CWE-PSS with V-Shaped pit features on top surface are investigated. The density of threading dislocations is found to be dramatically reduced from $1.28 \times 10^{9} / \mathrm{cm}^{2}$ to $3.62 \times 10^{8} / \mathrm{cm}^{2}$ since the stacking faults induced by CWE-PSS during the initial lateral growth of GaN effectively blocks them. Thus, a high internal quantum efficiency of $63 \%$ was achieved and this is measured by temperature-dependent PL intensity with variation of excitation laser power. The roughening surface consisted of $\mathrm{V}$-shaped pits can be thought of as a strong diffuser by using the paraboloidal autocorrelation function, and that leads to $20 \%$ enhancement in light extraction efficiency. Moreover, an additional enhancement of $7.8 \%$ in light extraction efficiency was obtained due to CWE-PSS that can effectively diffract guided light into escaped cones of LED chips. Thus, the combination of all of the above advantageous mechanisms provides a dramatic enhancement of $45 \%$ in the external quantum efficiency, and demonstrates that the CWE-PSS combined with V-shaped pits on top surface is a promising structure for next-generation lighting source.

\section{REFERENCES}

[1] S. Nakamura, S. Pearton, and G. Fasol, The Blue Laser Diode: The Complete Story, 2nd ed. Berlin, Germany: Springer, 2000.

[2] S. Nakamura, M. Senoh, S. Nagahama, N. Iwasa, T. Yamada, T. Matsushita, H. Kiyoku, Y. Sugimoto, T. Kozaki, H. Umemoto, M. Sano, and K. Chocho, "InGaN/GaN/AlGaN-based laser diodes with modulation-doped strained-layer superlattices grown on an epitaxially laterally overgrown GaN substrate," Appl. Phys. Lett., vol. 72, pp. 211-213, 1998.

[3] E. F. Schubert, Light-Emitting Diodes, Second ed. Cambridge, U.K.: Cambridge Univ. Press, 2006.

[4] M. Broditsky and E. Yablonovitch, "Light-emitting-diode extraction efficiency,” Proc. SPIE, vol. 3002, pp. 119-122, 1997.

[5] Z. H. Feng and K. M. Lau, "Enhanced luminescence from GaN-based blue LEDs grown on grooved sapphire substrates," IEEE Photon. Technol. Lett., vol. 17, no. 9, pp. 1812-1814, Sep. 2005.

[6] S. J. Chang, Y. K. Su, Y. C. Lin, R. W. Chuang, C. S. Chang, J. K. Sheu, T. C. Wen, S. C. Shei, C. W. Kuo, and D. H. Fan, "MOCVD growth of InGan/GaN blue light emitting diodes on patterned sapphire substrates," Phys. Stat. Sol. (C), vol. 7, pp. 2253-2256, 2003.

[7] D. S. Wuu, W. K. Wang, W. C. Shih, R. H. Horng, C. E. Lee, W. Y. Lin, and J. S. Fang, "Enhanced output power of near-ultraviolet InGaN-GaN LEDs grown on patterned sapphire substrates," IEEE Photon. Technol. Lett., vol. 17, no. 2, pp. 288-290, Feb. 2005.

[8] Y. J. Lee, T. C. Hsu, H. C. Kuo, S. C. Wang, Y. L. Yang, S. N. Yen, Y. T. Chu, Y. J. Shen, M. H. Hsieh, M. J. Jou, and B. J. Lee, "Improvement in light-output efficiency of near-ultraviolet InGaN-GaN LEDs fabricated on stripe patterned sapphire substrates," Mater. Sci. Eng., B, vol. 122 , no. 3, pp. 184-187, 2005. 
[9] K. Tadatomo, H. Okagawa, Y. Ohuchi, T. Tsunekawa, Y. Imada, M. Kato, and T. Tahuchi, "High output power InGaN ultraviolet lightemitting diodes fabricated on patterned substrates using metalorganic vapor phase epitaxy," Jpn. J. Appl. Phys., vol. 40, pp. L583-L585, 2001.

[10] T. Fujii, Y. Gao, R. Sharma, E. L. Hu, S. P. DenBaars, and S. Nakamura, "Increase in the extraction efficiency of GaN-based light-emitting diodes via surface roughening," Appl. Phys. Lett., vol. 84, pp. 855-857, 2004.

[11] J. K. Kim, H. Luo, Y. Xi, J. M. Shah, T. Gessmann, and E. F. Schubert, "Light extraction in GaInN light-emitting diodes using diffuse omnidirectional reflectors," J. Electrochem. Soc., vol. 153, pp. G105-G107, 2006.

[12] Y. J. Lee, T. C. Lu, H. C. Kuo, and S. C. Wang, "High light-extraction GaN-based LEDs with double diffuse surfaces," IEEE J. Quantum Electron., vol. 42, no. 12, pp. 1196-1201, Dec. 2006.

[13] Y. J. Lee, J. M. Hwang, T. C. Hsu, M. H. Hsieh, M. J. Jou, B. J. Lee, T. C. Lu, H. C. Kuo, and S. C. Wang, "Enhancing the output power of GaN-based LEDs grown on chemical wet etching patterned sapphire substrate," IEEE Photon. Technol. Lett., vol. 18, no. 10, pp. 1152-1154, May 2006.

[14] T. V. Cuong, H. S. Cheong, H. G. Kim, C.-H. Hong, E. K. Suh, H. K. Cho, and B. H. Kong, "Enhanced light output from aligned micropit InGaN-based light emitting diodes using wet-etch sapphire patterning," Appl. Phys. Lett., vol. 90, p. 131107, 2007.

[15] S. J. Chang, L. W. Wu, Y. K. Su, Y. P. Hsu, W. C. Lai, J. M. Tsai, J. K. Sheu, and C. T. Lee, "Nitride-based LEDs with 800/spl deg/C grown p-AlInGaN-GaN double-cap layers," IEEE Photon. Technol. Lett., vol. 16, no. 6, pp. 1447-1449, 2004.

[16] Y. Chen, T. Takeuchi, H. Amano, I. Akasaki, N. Yamada, Y. Kaneko, and S. Y. Wang, "Pit formation in GaInN quantum wells," Appl. Phys. Lett., vol. 72, pp. 710-712, 1998.

[17] I.-H. Kim, H.-S. Park, Y.-J. Park, and T. Kim, "Formation of V-shaped pits in InGaN/GaN multiquantum wells and bulk InGaN films," Appl. Phys. Lett., vol. 73, pp. 1634-1636, 1998.

[18] D. I. Florescu, S. M. Ting, J. C. Ramer, D. S. Lee, V. N. Merai, A. Parkeh, D. Lu, E. A. Armour, and L. Chernyak, "Investigation of V-defects and embedded inclusions in InGan/GaN multiple quantum wells grown by metalorganic chemical vapor deposition on (0001) sapphire," Appl. Phys. Lett., vol. 83, pp. 33-35, 2003.

[19] L. G. Shirley and N. George, "Diffuser radiation patterns over a large dynamic range," Appl. Opt., vol. 27, pp. 1850-1861, 1988.

[20] Z. H. Feng, Y. D. Qi, Z. D. Lu, and K. M. Lau, "GaN-based blue lightemitting diodes grown and fabricated on patterned sapphire substrates by metalorganic vapor-phase epitaxy," J. Cryst. Growth, vol. 272, pp. 327-332, 2004.

[21] H. K. Cho, J. Y. Lee, K. S. Kim, G. M. Yang, J. H. Song, and P. W. Yu, "Effect of buffer layers and stacking faults on the reduction of threading dislocation density in $\mathrm{GaN}$ overlayers grown by metalorganic chemical vapor deposition," J. Appl. Phys., vol. 89, pp. 2617-2621, 2001.

[22] S. Watanabe, N. Yamada, M. Nagashima, Y. Ueki, C. Sasaki, Y. Tamada, T. Taguchi, K. Tadatomo, H. Okagawa, and H. Kudo, "Internal quantum efficiency of highly-efficient $\operatorname{In}_{x} \mathrm{Ga}_{1-x} \mathrm{~N}$-based near-ultraviolet light-emitting diodes," Appl. Phys. Lett., vol. 83, pp. 4906-4908, 2003

[23] H. A. Haus, Waves and Fields in Optoelectronics. Englewood Cliffs, NJ: Prentice-Hall, p. 48.

[24] P. Visconti, K. M. Jones, M. A. Reshchikov, R. Cingolani, H. Morkoç, and R. J. Molnar, "Dislocation density in GaN determined by photoelectrochemical and hot-wet etching," Appl. Phys. Lett., vol. 77, pp. $3532-3524,2000$.

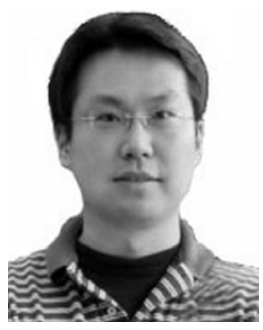

Ya-Ju Lee received the B.S. degree in physics from National Central University, Taiwan, R.O.C., in 2000 and the M.S. and Ph.D. degrees from the Institute of Electro-Optical Engineering, National Chiao Tung University, Taiwan, R.O.C., in 2002 and 2007, respectively.

He is currently a Postdoctoral Research Associate in Future Chips Constellation and Department of Physics, Applied Physics and Astronomy, Rensselaer Polytechnic Institute, Troy, NY.

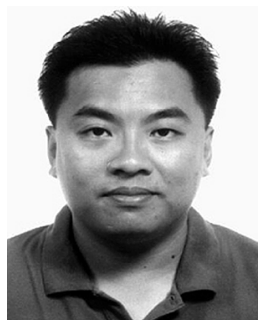

Hao-Chung Kuo (M'98-SM'06) received the B.S. degree in physics from National Taiwan University, Taiwan, R.O.C., the M.S. degree in electrical and computer engineering from Rutgers University, Piscataway, NJ, in 1995, and the Ph.D. degree from the Electrical and Computer Engineering Department of the University of Illinois-Urbana Champaign in 1999

$\mathrm{He}$ has an extensive professional career both in research and industrial research institutions that includes Research Consultant in Lucent Technologies, Bell Lab (1993-1995); a Senior Research Engineer at Filtronic Solid State (1999-2000); and a Member of Technical Staff in the Fiber-optics Division at Agilent Technologies (2000-2001) and LuxNet Corporation (2001-2002). Since October 2002, he joined National Chiao Tung University, Taiwan, R.O.C., as a Faculty Member of the Institute of Electro-Optical Engineering. His current research interests include semiconductor lasers, vertical-cavity surface-emitting lasers, blue and UV LED lasers, quantum confined optoelectronic structures, optoelectronic materials, and high-speed semiconductor devices. He has been an author and coauthor of 80 internal journal papers and four granted patents.

Dr. Kuo is member of The International Society for Optical Engineers (SPIE) and the Materials Research Society (MRS).

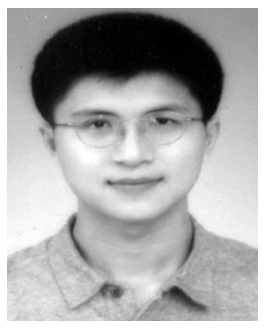

Tien-Chang Lu (M'07) received the B.S. degree in electrical engineering from National Taiwan University, Taiwan, R.O.C., in 1995, the M.S. degree in electrical engineering from the University of Southern California, Los Angeles, in 1998, and the $\mathrm{Ph} . \mathrm{D}$. degree in electrical engineering and computer science from National Chiao Tung University, Taiwan, R.O.C., in 2004.

He joined National Chiao Tung University as a Faculty Member of the Department of Photonics in August 2005

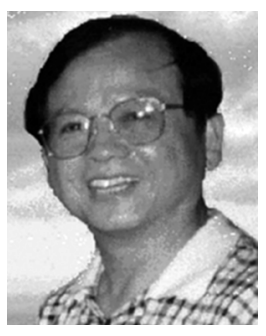

Shing-Chung Wang (M'79-SM'03-LM'07) received the B.S. degree from National Taiwan University, Taiwan, R.O.C., in 1957, the M.S. degree from National Tohoku University, Japan, in 1965, and the Ph.D. from Stanford University, Stanford, CA, in 1971, all in electrical engineering.

He has an extensive professional career both in academic and industrial research institutions that includes a Faculty Member at National Chiao Tung University, Taiwan, R.O.C. (1965-1967); a Research Associate at Stanford University (1971-1974); a Senior Research Scientist at Xerox Corporation (1974-1985); and a Consulting Scientist at Lockheed-Martin Palo Alto Research Laboratories (1985-1995). Since 1995, he rejoined National Chiao Tung University as a Faculty Member of the Institute of Electro-Optical Engineering. His current research interests include semiconductor lasers, vertical-cavity surface-emitting lasers, blue and UV lasers, quantum confined optoelectronic structures, optoelectronic materials, diode-pumped lasers, and semiconductor laser applications.

Prof. Wang is a Fellow of the Optical Society of America (OSA) and a recipient of Outstanding Scholar Award from the Foundation for the Advancement of Outstanding Scholarship.

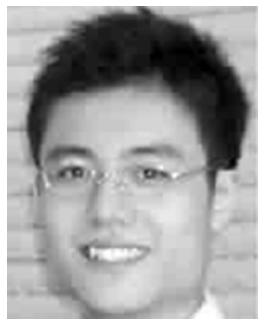

Kar Wei Ng received the B.Eng. and M.Phil. degrees in electronics engineering from the Hong Kong University of Science and Technology (HKUST) in 2004 and 2007, respectively. During his Master's studies, he was mainly involved with MOCVD growth and characterization of GaN-based light emitting diodes (LEDs). In particular, he focused on patterned growth on sapphire substrate engraved by wet etching as well as development of in situ surface texturing techniques.

Since May 2007, he has been a Research Assistant at HKUST, where he concentrates on the investigation of green LED degradation. 


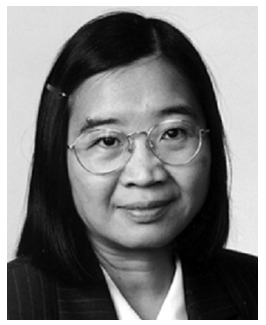

Kei May Lau (F'01) received the B.S. and M.S. degrees in physics from the University of Minnesota, MN, in 1976 and 1977, respectively, and the Ph.D. degree in electrical engineering from Rice University, Houston, TX, in 1981

She initiated metal-organic-chemical-vapor deposition (MOCVD), compound semiconductor materials, and devices programs at the University of Massachusetts/Amherst (UMass), where she became a Full Professor in 1993. Since July 2005, she became a Chair Professor of Electrical and Computer Engineering at the Hong Kong University of Science and Technology (HKUST), Hong Kong. Her research group performed studies on heterostructures, quantum wells, strained-layers, III-V selective epitaxy, as well as high-frequency and photonic devices.

Prof. Lau is a recipient of the National Science Foundation (NSF) Faculty Awards for Women (FAW) Scientists and Engineers. She served on the IEEE Electron Devices Society Administrative Committee and was an Editor of the IEEE TRAnsactions on EleCtron DevicEs from 1996 to 2002. She also served on the Electronic Materials Committee of the Minerals, Metals and Materials Society (TMS) of the American Institute of Materials Engineers (AIME).

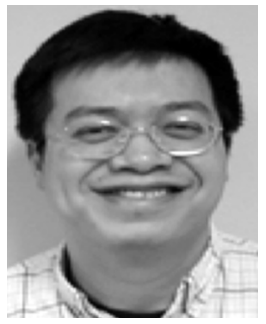

Zu-Po Yang received both the B.S. and M.S. degrees in physics from Nation Cheng Kung University, Taiwan, R.O.C., in 1998 and 2000, respectively. He is currently working towards the Ph.D. degree.

He works with Prof. S. Lin in the Physics Department and Future Chips Constellation of Rensselaer Polytechnic Institute, Troy, NY. His current research interests include optics measurement and nanofabrication.
Allan Shih-Ping Chang received the B.E. degree from the University of Melbourne, Australia, in 1996, the M.S. degree in electrical engineering from the University of Michigan, Ann Arbor, in 1998, and the Ph.D. degree in electrical engineering from Princeton University, Princeton, NJ, in 2005.

From 2005 to 2007, he was a Postdoctoral Research Associate in the Physics Department of Rensselaer Polytechnic Institute, Troy, NY. He is now with the Molecular Foundry, Materials Science Division, Lawrence Berkeley National Laboratory, Berkeley, CA.

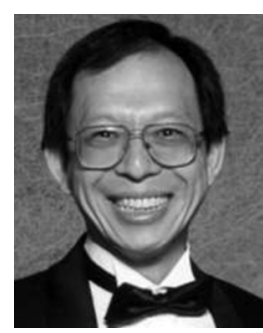

Shawn-Yu Lin received the Bachelor's degree from National Taiwan University, Taiwan, R.O.C., the Master's degree from the University of North Carolina-Chapel Hill, and Ph.D. degree from Princeton University, Princeton, NJ.

In 1992, he joined IBM T. J. Watson research center, first working on the wave-function symmetry of high-temperature superconductors and then on ultrafast photo-conductive switches. In 1994, he joined Sandia National Laboratory and led its efforts in developing photonic-crystal devices for communication, defense, and energy applications. In summer 2004, he was appointed as a Distinguished Professor at the Future-Chips Constellation and Department of Physics of Rensselaer Polytechnic Institute, Troy, NY. His current research interest is in active photonic crystal structures for sensing, beam steering, solid-state lighting, photon recycling, and solar energy applications. 